\title{
Preclinical Evaluation of Podoplanin-Targeted Alpha-Radioimmunotherapy with The Novel Antibody NZ-16 for Malignant Mesothelioma
}

\section{Hitomi Sudo}

QST NIRS: Kokuritsu Kenkyu Kaihatsu Hojin Ryoshi Kagaku Gijutsu Kenkyu Kaihatsu Kiko Hoshasen Igaku Sogo Kenkyujo

Atsushi B Tsuji ( $\nabla$ tsuji.atsushi@qst.go.jp )

National Institute of Radiological Sciences https://orcid.org/0000-0003-2726-288X

\section{Aya Sugyo}

QST NIRS: Kokuritsu Kenkyu Kaihatsu Hojin Ryoshi Kagaku Gijutsu Kenkyu Kaihatsu Kiko Hoshasen Igaku Sogo Kenkyujo

Mika K. Kaneko

Tohoku University: Tohoku Daigaku

\section{Yukinari Kato}

Tohoku University School of Medicine: Tohoku Daigaku Daigakuin Igakukei Kenkyuka Igakubu

\section{Kotaro Nagatsu}

QST: Kokuritsu Kenkyu Kaihatsu Hojin Ryoshi Kagaku Gijutsu Kenkyu Kaihatsu Kiko

Hisashi Suzuki

QST: Kokuritsu Kenkyu Kaihatsu Hojin Ryoshi Kagaku Gijutsu Kenkyu Kaihatsu Kiko

\section{Tatsuya Higashi}

QST: Kokuritsu Kenkyu Kaihatsu Hojin Ryoshi Kagaku Gijutsu Kenkyu Kaihatsu Kiko

\section{Research Article}

Keywords: molecular radiotherapy, dosimetry, relative biological effectiveness, actinium- 225, yttrium-90

Posted Date: July 12th, 2021

DOI: https://doi.org/10.21203/rs.3.rs-691419/v1

License: (c) (1) This work is licensed under a Creative Commons Attribution 4.0 International License. Read Full License 


\section{Preclinical evaluation of podoplanin-targeted alpha-radioimmunotherapy with the novel antibody NZ-16 for malignant mesothelioma}

Hitomi Sudo ${ }^{1}$, Atsushi B. Tsuji ${ }^{1}$, Aya Sugyo ${ }^{1}$, Mika K. Kaneko ${ }^{2}$, Yukinari Kato ${ }^{2,3}$, Kotaro Nagatsu $^{4}$, Hisashi Suzuki ${ }^{4}$, and Tatsuya Higashi ${ }^{1}$

\footnotetext{
${ }^{1}$ Department of Molecular Imaging and Theranostics, iQMS, National Institutes for Quantum and Radiological Science and Technology (QST), 4-9-1 Anagawa, Inage, Chiba 263-8555, Japan

${ }^{2}$ Department of Antibody Drug Development, Tohoku University Graduate School of Medicine, 2-1, Seiryo-machi, Aoba-ku, Sendai, Miyagi 980-8575, Japan ${ }^{3}$ Department of Molecular Pharmacology, Tohoku University Graduate School of Medicine, 2-1 Seiryo-machi, Aoba-ku, Sendai, Miyagi 980-8575, Japan ${ }^{4}$ Department of Advanced Nuclear Medicine Science, iQMS, National Institutes for Quantum and Radiological Science and Technology (QST), 4-9-1 Anagawa, Inage, Chiba 263-8555, Japan
}

Correspondence: AB Tsuji, Telephone: +81-43-382-3704; Fax: +81-43-206-0818; E-mail: tsuji.atsushi@qst.go.jp

\section{Word count of the manuscript (including references): 5,808}

Funding: This work was supported in part by KAKENHI 18K07778 (HS), 21K07688 (HS), $21 \mathrm{~K} 07230$ (AS), $18 \mathrm{H} 02774$ (ABT), 21K07168 (MKK), and 19K07705 (YK), and in part by the Japan Agency for Medical Research and Development (AMED) under Grant Numbers JP21 am0401013 (YK) and JP21 am0101078 (YK).

Conflicts of interest: The authors declare no conflict of interest for this article.

Ethics approval: The animal experimental protocol was approved by the Animal Care and Use Committee of the National Institutes for Quantum and Radiological Science and Technology (13-1022, 26 May 2016), and all animal experiments were conducted according to the institutional guidelines regarding animal care and handling.

Consent to participate: Not applicable. 
Consent for publication: Not applicable.

\begin{abstract}
Purpose This study aimed to evaluate the potential of podoplanin (PDPN)-targeted alpharadiotherapy (RIT) for treating malignant mesothelioma.
\end{abstract}

Methods A newly developed anti-PDPN antibody, NZ-16, and a previous anti-PDPN antibody, NZ-12, were assessed. The in vitro properties of radiolabeled antibodies were evaluated by cell binding and competitive inhibition assays using PDPN-expressing NCI-H226 (H226) mesothelioma cells. The biodistribution of ${ }^{111} \mathrm{In}$-labeled antibodies was studied in tumorbearing mice. Tumor volumes and body weights of mice treated with ${ }^{90} \mathrm{Y}$ - and ${ }^{225} \mathrm{Ac}-$ labeled NZ-16 were measured for 56 days. The absorbed doses were estimated on the basis of the biodistribution data. Pathologic analysis of tumors and organs was conducted.

Results The radiolabeled NZ-16 specifically bound to H226 cells with higher affinity than NZ12. The biodistribution studies showed higher tumor uptake of radiolabeled NZ-16 compared with NZ-12. RIT with ${ }^{225}$ Ac-labeled NZ-16 (11.1 and $\left.18.5 \mathrm{kBq}\right)$ had a significantly higher antitumor effect than RIT with ${ }^{90}$ Y-labeled NZ-16 (3.7 MBq; $\left.P<0.01\right)$. ${ }^{225}$ Ac-labeled NZ-16 induced more necrosis compared with ${ }^{90}$ Y-labeled NZ-16, but the Ki-67 index and apoptosis rate were similar. The estimated absorbed doses were expected to be tolerable in mice. Temporary body weight loss occurred, but recovered within several days. No visible damage to major organs was detected.

Conclusion The novel anti-PDPN antibody NZ-16 was a more effective RIT agent than NZ12. Radiolabeled NZ-16, especially ${ }^{225}$ Ac-labeled NZ-16, markedly suppressed tumor growth and prolonged survival without inducing severe adverse effects. RIT with radiolabeled NZ-16 is a promising therapeutic option for malignant mesothelioma.

Keywords: molecular radiotherapy; dosimetry; relative biological effectiveness; actinium225; yttrium-90 


\section{Introduction}

Malignant mesothelioma is an aggressive tumor that arises primarily in the pleural or peritoneal mesothelial surfaces [1]. Surgical resection is only offered to patients with early-stage disease $[1,2]$. Most patients reach advanced-stage disease before diagnosis, and thus the primary treatment is systemic chemotherapy $[1,2]$. The prognosis is poor and the median overall survival of patients who undergo chemotherapy is approximately 12 months [2]. Therefore, the development of more effective treatments for unresectable malignant mesothelioma is strongly desired.

Podoplanin (PDPN), which is overexpressed in more than $80 \%$ of malignant mesothelioma, is a promising therapeutic target [3]. PDPN is a type I transmembrane sialomucin-like glycoprotein expressed in kidney podocytes, alveolar type I cells, and lymphatic endothelial cells [4]. High expression of PDPN in tumors is associated with epithelial mesenchymal transition, migration, invasion, and metastasis [5, 6]. Several preclinical studies have demonstrated that anti-PDPN antibodies inhibit cancer metastasis [7] and cancer progression $[8,9]$. Therefore, PDPN is a promising therapeutic target for malignant mesothelioma.

Radioimmunotherapy (RIT) is a selective internal radiation therapy in which highaffinity antibodies against tumor-associated antigens are used to transport radionuclides to tumors [10]. In clinical practice, RIT for hematologic malignancies such as non-Hodgkin's lymphoma utilizes anti-CD20 antibodies conjugated with $\beta$-emitters, ${ }^{90} \mathrm{Y}$ or ${ }^{131} \mathrm{I}$, and the overall response rates are high, reaching $60 \%-80 \%$, with a complete remission rate of $15 \%-$ $40 \%[10,11]$. The clinical efficacy of existing RIT for solid tumors, however, remains low, mainly due to the low radiosensitivity of solid tumors. Overcoming the radioresistance is necessary to enhance the clinical efficacy of RIT.

The clinical efficacy of $\alpha$-particle emitters in the treatment of solid cancer was recently demonstrated [12]. $\alpha$-Particle emitters have a greater linear energy transfer compared with $\beta$ emitters and deposit more energy into tumor cells, which results in greater DNA damage to the cells [13]. Actinium-225 is an $\alpha$-particle-emitting radionuclide that generates a total of $4 \alpha$ particles in the decay chain [14]. The half-life of ${ }^{225} \mathrm{Ac}$ is appropriate for the pharmacokinetics of antibodies. Therefore, RIT with ${ }^{225} \mathrm{Ac}$ is expected to improve the therapeutic efficacy of RIT treatment for solid tumors. 
A previous study reported that ${ }^{90}$ Y-labeled anti-PDPN antibody NZ-12 suppresses tumor growth in a mesothelioma model cell line NCI-H226 (H226); unfortunately, complete remission was not achieved [15]. To improve the therapeutic effect of RIT with an anti-PDPN antibody, we newly developed an anti-PDPN antibody, NZ-16, having a different constant region than NZ-12. NZ-16 has a higher affinity than NZ-12 for H226 mesothelioma cells and is therefore expected to deliver more radionuclides to the tumors. In the present study, we first compared the in vitro and in vivo properties of NZ-12 and NZ-16 radiolabeled with ${ }^{111} \mathrm{In}$. After confirming that NZ-16 has more favorable binding properties than NZ-12, the antitumor effects of ${ }^{225}$ Ac-labeled NZ-16 were compared with those of ${ }^{90}$ Y-labeled NZ-16 in an H226 mesothelioma mouse model. 


\section{Materials and methods}

\section{Antibody}

A rat-human chimeric anti-human PDPN antibody, NZ-12, was previously generated [16]. To generate the novel chimeric anti-human PDPN antibody NZ-16, the appropriate heavy chain variable domain of a rat NZ-1 antibody [17] and heavy chain constant domain of human $\operatorname{IgG}_{1}$ were subcloned into the pCAG-Neo vector (FUJIFILM Wako Pure Chemical Corporation, Osaka, Japan), and the light chain variable domain of a rat NZ-1 antibody and human lambda light chain constant domain were subcloned into pCAG-Ble vectors (FUJIFILM Wako Pure Chemical Corporation). The vectors were transfected into ExpiCHO-S cells using the ExpiCHO Expression System (Thermo Fisher Scientific Inc., Waltham, MA, USA). NZ-16 was purified using Protein G-Sepharose (GE Healthcare Bio-Sciences, Pittsburgh, PA, USA).

\section{Cell culture}

Mesothelioma cell line NCI-H226 (H226, CRL-5826) was obtained from ATCC (Manassas, VA, USA). The cells were cultured in RPMI-1640 (FUJIFILM Wako Pure Chemical Corporation) containing 10\% fetal bovine serum (Thermo Fisher Scientific Inc.) in $5 \% \mathrm{CO}_{2}$ at $37^{\circ} \mathrm{C}$

\section{Radiolabeling of antibodies}

Antibodies were conjugated with $p$-SCN-Bn-DOTA (DOTA, Macrocyclics, Dallas, TX, USA) as described previously [18]. Briefly, antibodies $(5 \mathrm{mg} / \mathrm{mL})$ were reacted with 4 equal molar amounts of DOTA in $50 \mathrm{mM}$ borate buffer $(\mathrm{pH} 8.5)$ for $16 \mathrm{~h}$ at $37^{\circ} \mathrm{C}$. The conjugation ratios of DOTA to antibodies were estimated to be approximately 2.8 each as determined by radio-thin layer chromatography with $80 \%$ methanol. The DOTA-conjugated antibodies were purified by elution with 0.1 M acetate buffer ( $\mathrm{pH}$ 6.0) using a Sephadex G-50 column (GE Healthcare BioSciences). ${ }^{111} \mathrm{InCl}_{3}$ (Nihon Medi-Physics, Tokyo, Japan) or ${ }^{90} \mathrm{YCl}_{3}$ (Perkin Elmer, Waltham, MA, USA) was incubated in $0.5 \mathrm{M}$ acetate buffer ( $\mathrm{pH}$ 6.0) for $5 \mathrm{~min}$ at room temperature. Each was mixed with the DOTA-antibody conjugate and incubated for $60 \mathrm{~min}$ at $37^{\circ} \mathrm{C}$. Radiolabeling of the antibody with ${ }^{225} \mathrm{Ac}$ was conducted as described previously [18]. ${ }^{225} \mathrm{AcNO}_{3}$ (Oak Ridge National Laboratory, Oak Ridge, TN, USA) dissolved in $0.2 \mathrm{M}$ optima 
grade $\mathrm{HCl}$ (Thermo Fisher Scientific Inc.) was added to $2 \mathrm{M}$ tetramethylammonium acetate (Tokyo Chemical Industry, Tokyo, Japan) and 150g/L L-ascorbic acid (MilliporeSigma, St. Louis, MO, USA), and the solution was incubated for $5 \mathrm{~min}$ at room temperature. The solution was then mixed with the DOTA-conjugated antibodies and incubated for $60 \mathrm{~min}$ at $37^{\circ} \mathrm{C}$. The radiolabeled antibodies were purified using an Amicon Ultra centrifugal filter (Merck Millipore, Darmstadt, Germany), and the purified antibodies were analyzed by radio-thin layer chromatography. The specific activity of ${ }^{111}$ In-labeled NZ-12, ${ }^{111}$ In-labeled NZ-16, ${ }^{90}$ Y-labeled NZ-16, and ${ }^{225}$ Ac-labeled NZ-16 was approximately $4.9 \pm 2.5,9.7 \pm 4.5,662.3 \pm 151.8$, and $0.6 \pm 0.1 \mathrm{kBq} / \mu \mathrm{g}$, respectively. The radiochemical yield was approximately $40 \%$ for ${ }^{111} \mathrm{In}$ labeled NZ-12, 50\%-80\% for ${ }^{111}$ In-labeled NZ-16, $90 \%$ for ${ }^{90}$ Y-labeled NZ-16, and $25 \%$ for ${ }^{225}$ Ac-labeled NZ-16. The radiochemical purities were greater than $95 \%$ after purification.

\section{Cell binding and competitive inhibition assays}

For the cell binding assays, H226 cells $\left(1.0 \times 10^{7}, 5.0 \times 10^{6}, 2.5 \times 10^{6}, 1.3 \times 10^{6}, 6.3 \times 10^{5}, 3.1\right.$ $\times 10^{5}, 1.6 \times 10^{5}$, and $7.8 \times 10^{4}$ ) in phosphate-buffered saline with $1 \%$ bovine serum albumin (MilliporeSigma) were incubated with ${ }^{111}$ In-labeled NZ-12 or NZ-16 antibodies on ice for 60 min. After washing, cell-bound radioactivity was measured using a gamma counter (Wizard2 Automatic Gamma Counters, PerkinElmer, Waltham, MA, USA). For competitive inhibition assays, $\mathrm{H} 226$ cells $\left(1.0 \times 10^{6}\right)$ in phosphate-buffered saline with $1 \%$ bovine serum albumin were incubated with ${ }^{111}$ In-labeled NZ-12 or NZ-16 in the presence of varying concentrations of intact NZ-12, intact NZ-16, DOTA-conjugated NZ-12, or DOTA-conjugated NZ-16 antibodies $(0,0.02,0.07,0.2,0.7,2.0,6.1,18.2$, and $54.5 \mathrm{nmol} / \mathrm{L})$ on ice for $60 \mathrm{~min}$. After washing, cell-bound radioactivity was measured with a gamma counter. The dissociation constant was estimated by applying data to a one-site competitive binding model using GraphPad Prism 8 software (GraphPad Software, La Jolla, CA, USA).

\section{Tumor model}

The animal experimental protocol was approved by the Animal Care and Use Committee of the National Institutes for Quantum and Radiological Science and Technology (13-1022, 26 May 2016), and all animal experiments were conducted according to the institutional 
guidelines regarding animal care and handling. H226 cells $\left(5 \times 10^{6}\right)$ were subcutaneously inoculated into male nude mice (BALB/c-nu/nu, 4 weeks old, CLEA Japan, Tokyo, Japan) under isoflurane anesthesia.

\section{Biodistribution of radiolabeled antibodies}

When tumor volumes reached approximately $50 \mathrm{~mm}^{3}$, mice ( $\mathrm{n}=4$-5/time-point), were intravenously injected with ${ }^{111}$ In-labeled NZ-12 (37 kBq), ${ }^{111}$ In-labeled NZ-16 (37 kBq), or ${ }^{225}$ Ac-labeled NZ-16 $(3.7 \mathrm{kBq})$ in a total of $40 \mu \mathrm{g}$ antibody adjusted by adding the corresponding unlabeled antibodies. The mice were killed by isoflurane inhalation at 1, 2, 4, or 7 days after injecting the ${ }^{111} \mathrm{In}$-labeled antibodies and 4 days after injecting the ${ }^{225} \mathrm{Ac}-$ labeled NZ-16. Blood was obtained from the heart, and the tumor, brain, liver, spleen, intestine, kidney, and muscle were dissected and weighed. Radioactivity was measured using a gamma counter with an energy window of $150-350 \mathrm{keV}$ for ${ }^{111}$ In and $200-300 \mathrm{keV}$ for ${ }^{225}$ Ac. The uptake is represented as a percentage of the injected dose (radioactivity) per gram of tissue (\% ID/g).

\section{Dosimetry}

As described previously [19], the absorbed doses of the ${ }^{90} \mathrm{Y}$ - and ${ }^{225} \mathrm{Ac}-$ labeled antibodies were estimated using the area under the curve based on the biodistribution data of the ${ }^{111} \mathrm{In}$-labeled antibodies and the mean energy emitted per transition of Y-90, $1.495 \times 10^{-13} \mathrm{~Gy} \mathrm{~kg}(\mathrm{~Bq} \mathrm{~s})^{-1}$ [20] and that of Ac-225 and all the daughter nuclei with corrections for branching, $4.6262 \times$ $10^{-12} \mathrm{~Gy} \mathrm{~kg}(\mathrm{~Bq} \mathrm{~s})^{-1}$ [20]. The absorbed dose of bone marrow was based on the blood data, considering a red-marrow-to-blood activity ratio of 0.4 [21]. Radiation weighting factors of 1 and 5 were used for Y-90 and Ac-225, respectively, as recommended by the Medical Internal Radiation Dose Committee [22]. The estimated absorbed dose is expressed as Sv when considering the radiation weighting factors.

\section{Radioimmunotherapy with ${ }^{90} \mathrm{Y}$ - and ${ }^{225}$ Ac-labeled antibody}

The mice were intravenously injected with intact NZ-16 (0 MBq, $\mathrm{n}=5),{ }^{90}$ Y-labeled NZ-16 (3.7 MBq, $\mathrm{n}=5)$, or ${ }^{225}$ Ac-labeled NZ-16 $(11,1$ and $18.5 \mathrm{kBq}, \mathrm{n}=5)$ antibodies at a total of 40 $\mu \mathrm{g}$ antibody adjusted by adding the corresponding unlabeled antibodies. Tumor sizes and body 
weights were measured at least twice a week for 8 weeks after administration. Tumor size was measured using a digital caliper, and tumor volume was calculated according to the following formula: tumor volume $\left(\mathrm{mm}^{3}\right)=\left(\right.$ length $\times$ width $\left.^{2}\right) / 2$. When the tumor volume reached greater than $800 \mathrm{~mm}^{3}$ or body weight loss was more than $20 \%$ compared with that at day 0 , the mouse was killed humanely by isoflurane inhalation.

\section{Histologic analysis}

H226 tumors were resected from mice on days 1,3 , and 7 after injection with intact NZ-16 (0 MBq, $\mathrm{n}=3$ /time-point), ${ }^{90}$ Y-labeled NZ-16 (3.7 MBq, $\mathrm{n}=3 /$ time-point), or ${ }^{225}$ Ac-labeled NZ$16(18.5 \mathrm{kBq}, \mathrm{n}=3 /$ time-point $)$. The tumors were fixed in $10 \%$ neutral-buffered formalin and embedded in paraffin. The tumor sections $(1-\mu \mathrm{m}$ thick) were deparaffinized and stained with hematoxylin and eosin. Tumor cell proliferation was evaluated by Ki-67-immunohistochemical staining with a rabbit anti-Ki-67 antibody (SP6, Abcam, Cambridge, MA, USA) and an antirabbit HRP/DAB Detection kit (Abcam) according to the manufacturer's instructions. The Ki67 index was calculated by counting the percentage of Ki-67-positive tumor cells per $>2500$ tumor cells in a section with $200 \times$ magnification $(n=3)$.

\section{Statistical Analysis}

Data are expressed as the means \pm standard deviation. Statistical analysis was performed using GraphPad Prism 8 software. Cell binding data and tumor volume data were analyzed by 2-way ANOVA. Ki-67 staining data were analyzed by 1-way ANOVA with Tukey's multiple comparison post-hoc test. Uptake data of radiolabeled antibodies were analyzed by unpaired $t$ test. Log-rank tests were used to evaluate Kaplan-Meier survival curves based on a tumor volume endpoint of $300 \mathrm{~mm}^{3} . P<0.05$ was considered statistically significant in all experiments. 


\section{Results}

\section{In vitro characterization of the antibodies}

In the cell binding assays with H226, ${ }^{111}$ In-labeled NZ-16 showed significantly higher specific binding than ${ }^{111}$ In-labeled NZ-12 $(P<0.01)$. The maximum values were $29.6 \pm 3.7 \%$ for NZ16 at $1.0 \times 10^{7}$ cells and $22.9 \pm 3.3 \%$ for NZ-12 at $1.0 \times 10^{7}$ (Fig. 1a and $1 \mathrm{~b}$ ). The specific binding did not significantly differ between ${ }^{111}$ In- and ${ }^{225}$ Ac-labeled NZ-16 (Supplementary Fig. 1). The results of the competitive inhibition assay are shown in Figures 1c and 1d. The binding affinities $\left(\mathrm{K}_{\mathrm{d}}\right)$ of intact NZ-12 and DOTA-conjugated NZ-12 were estimated to be 1.7 and $5.7 \mathrm{nM}$, respectively (Fig. 1c). Those of intact NZ-16 and DOTA-conjugated NZ-16 were estimated to be 1.8 and $2.7 \mathrm{nM}$, respectively (Fig. 1d). These results indicate that the DOTA conjugation procedure decreased the affinity of NZ-16 for PDPN to a lesser extent than that of NZ-12.

\section{Biodistribution of ${ }^{111}$ In-labeled antibodies in nude mice bearing $\mathrm{H} 226$ tumors}

The biodistribution of ${ }^{111}$ In-labeled NZ-12 and NZ-16 in H226 tumor-bearing mice is shown in Table 1 and Figure 2. ${ }^{111}$ In-Labeled NZ-12 and NZ-16 gradually cleared from the blood and accumulated in the H226 tumors. The uptake of ${ }^{111}$ In-labeled NZ-16 in normal organs tended to be higher than that of ${ }^{111}$ In-labeled NZ-12, except in the liver (Table 1). The uptake of ${ }^{111} \mathrm{In}$ labeled NZ-16 in the liver was significantly lower than that of NZ-12 on days 1 and 2 after injection $(P<0.05$ for day $1, P<0.01$ for day 2$)$. The uptake of ${ }^{225} \mathrm{Ac}-\mathrm{NZ}-16$ in normal organs was significantly higher than that of ${ }^{111}$ In-labeled NZ-16 $(P<0.01$ in the brain, lung, spleen, and muscle, $P<0.05$ in the intestine, Table 2).

Tumor uptake of ${ }^{111}$ In-labeled NZ-16 tended to be higher than that of NZ-12 over the observation period (Fig. 2). The maximal tumor uptake of ${ }^{111}$ In-labeled NZ-16 was significantly higher than that of ${ }^{111}$ In-labeled NZ-12 at day 4 after injection $(15.1 \pm 2.4 \% \mathrm{ID} / \mathrm{g}$ for NZ-16 and $10.0 \pm 0.4 \%$ ID/g for NZ-12; $P<0.01$, Fig. 2). The tumor uptake of ${ }^{225} \mathrm{Ac}-\mathrm{NZ}-$ 16 at day 4 was higher than that of ${ }^{111}$ In-labeled NZ-12 and NZ-16 $(P<0.01$, Fig. 2$)$.

\section{Dosimetry}

The absorbed doses were estimated on the basis of the biodistribution studies when In-111 was 
replaced with Y-90 or Ac-225. Table 3 shows the estimated absorbed doses when the radiationweighted factor was not considered. The absorbed doses of radiolabeled NZ-16 for tumors and organs tended to be higher than those of radiolabeled NZ-12, except for in the liver and kidney, although the difference between the 2 antibodies was not statistically significant (Table 3). The tumor-absorbed doses of ${ }^{225} \mathrm{Ac}$-labeled NZ-12 and NZ-16 were 60 -fold greater than those of ${ }^{90}$ Y-labeled NZ-12 and NZ-16, respectively (Table 3).

The relative biologic effect (RBE) was determined by calculating the absorbed doses from treatments with ${ }^{90} \mathrm{Y}$ - and ${ }^{225} \mathrm{Ac}-$ labeled NZ-16 without considering the radiation-weighted factor (Table 4). The dose absorbed by tumors treated with $3.7 \mathrm{kBq}$ of ${ }^{90}$ Y-labeled NZ-16 was 5.7-fold higher than that of tumors treated with $11.1 \mathrm{kBq}$ of ${ }^{225} \mathrm{Ac}-\mathrm{labeled}$ NZ-16 (Table 4).

With regard to safety, the absorbed doses considering radiation weighting factors of 1 for ${ }^{90} \mathrm{Y}$ and 5 for ${ }^{225} \mathrm{Ac}$ are shown in Table 5. The absorbed dose to bone marrow from treatment with $3.7 \mathrm{MBq}$ of ${ }^{90} \mathrm{Y}-$ labeled NZ-16 was higher than that from treatment with $11.1 \mathrm{kBq}$ of ${ }^{225} \mathrm{Ac}-$ labeled NZ-16 (Table 5). The absorbed doses to tumors and organs injected with $18.5 \mathrm{kBq}$ of ${ }^{225}$ Ac-labeled NZ-16 were higher than those of tumors injected with $3.7 \mathrm{MBq}$ of ${ }^{90}$ Y-labeled NZ-16 (Table 5).

\section{Treatment effects of radiolabeled antibodies in nude mice bearing $\mathrm{H226}$ tumors}

Marked antitumor effects were observed in mice treated with ${ }^{90} \mathrm{Y}$ - and ${ }^{225}$ Ac-labeled NZ-16 ( $P$ $<0.01$, vs. $0 \mathrm{MBq}$, Fig. 3a). Treatment with $3.7 \mathrm{MBq}$ of ${ }^{90} \mathrm{Y}-\mathrm{NZ}-16$ reduced tumor volume from day 7 to day 21 after injection, and thereafter the tumor volume gradually increased $(P<$ 0.01 vs. $0 \mathrm{MBq}$, Fig. 3a). In the group injected with $11.1 \mathrm{kBq}$ of ${ }^{225} \mathrm{Ac}-\mathrm{NZ}-16$, the tumor volume increased during the first 10 days, and thereafter decreased until day $42(P<0.01$ vs. $0 \mathrm{MBq}$, Fig. 3a). In the group injected with $18.5 \mathrm{kBq}$ of ${ }^{225} \mathrm{Ac}-\mathrm{NZ}-16$, tumor growth was suppressed during the first 28 days, and thereafter the tumor volume decreased until the end of the observation period $\left(P<0.01\right.$ vs. $0 \mathrm{MBq}$ and 3.7 MBq of ${ }^{90}$ Y-NZ-16, Fig. 3a).

Kaplan-Meier survival curves based on a tumor volume endpoint of $300 \mathrm{~mm}^{3}$ are shown in Figure 3b. Injection with $3.7 \mathrm{MBq}$ of ${ }^{90}$ Y-labeled NZ-16, $11.1 \mathrm{kBq}{ }^{225}$ Ac-labeled NZ-16, and $18.5 \mathrm{kBq}$ of ${ }^{225} \mathrm{Ac}-$ labeled NZ-16 significantly prolonged survival compared with the $0-\mathrm{MBq}$ groups $(P<0.01)$. At the end of the observation period, all mice treated with $11.1 \mathrm{kBq}$ and 18.5 
$\mathrm{kBq}$ of ${ }^{225} \mathrm{Ac}-\mathrm{NZ}-16$ were defined as surviving, and survival in the ${ }^{90} \mathrm{Y}$-treatment group was $60 \%$. No statistically significant difference in survival was detected among the 3 groups treated with the radiolabeled antibodies (Fig. 3b).

The 3 radiolabeled treatments induced temporary weight loss. Body weight loss never exceeded $20 \%$ compared with that at day 0 , however, which is the criterion for humane euthanasia (Supplementary Fig. 2a). No obvious damage was detected in the spleen, kidney, liver, or bone marrow in mice treated with ${ }^{90} \mathrm{Y}-$ or ${ }^{225}$ Ac-labeled NZ-16 (Supplementary Fig. 2b)

\section{Histologic analysis of $\mathrm{H} 226$ tumors treated with ${ }^{90}$ Y-labeled NZ-16}

Tumors treated with $0 \mathrm{MBq}$ of NZ-16 (intact NZ-16 only) were composed of solid nests of epithelial cells and some mitotic cells (Fig. 4 upper panels). Sections of H226 tumors treated with 3.7 MBq of ${ }^{90}$ Y-labeled NZ-16 showed a few small necrotic foci on day 1, and expansion of the necrotic area was observed on days 3 and 7 (Fig. 4 middle panels). In the tumors treated with $18.5 \mathrm{kBq}$ of ${ }^{225} \mathrm{Ac}-\mathrm{NZ}-16$ on day 1 , more necrotic foci were observed compared with the tumors treated with ${ }^{90} \mathrm{Y}-\mathrm{NZ}-16$, and extensive necrosis and lymphocyte infiltration were observed on day 3 after injection (Fig. 4, lower panels). On day 7, the tumor cells decreased and partial replacement of necrotic tumor cells by fibrous tissue was observed in ${ }^{225}$ Ac-NZ-16treated tumors (Fig. 4, lower panels).

Treatment with $3.7 \mathrm{MBq}$ of ${ }^{90}$ Y-labeled NZ-16 and $18.5 \mathrm{kBq}$ of ${ }^{225}$ Ac-labeled NZ-16 significantly reduced the proliferation of (Ki-67-positive) tumor cells compared with tumors treated with $0 \mathrm{MBq}$ NZ-16 (intact NZ-16) on days $1-7$ after injection $(P<0.01$, Fig. 5a and 5b). A few apoptotic cells were observed in tumors treated with ${ }^{90} \mathrm{Y}-$ and ${ }^{225} \mathrm{Ac}-\mathrm{NZ}-16$, but no apoptosis was observed in the 0-MBq group (Supplementary Fig. 3). 


\section{Discussion}

An anti-PDPN antibody, NZ-16, was newly generated from the parental antibody NZ-1. The constant region of the NZ-16 heavy chain differs from that in NZ-12. NZ-16 has a higher affinity and showed higher tumor uptake in a PDPN-expressing H226 mesothelioma mouse model. Therefore, RIT with NZ-16 was expected to be more effective and NZ-16 was selected for further evaluation. As expected, ${ }^{90} \mathrm{Y}$ - and ${ }^{225} \mathrm{Ac}-$ labeled NZ-16 showed significant antitumor effects in tumor-bearing mice, compared with ${ }^{90}$ Y-labeled NZ-12 [15]. The significantly higher effectiveness of ${ }^{225}$ Ac-labeled NZ-16 compared with ${ }^{90}$ Y-labeled NZ-16 $(P<0.01)$ suggests that $\alpha$-RIT with NZ-16 is a promising therapy for malignant mesothelioma. PDPN is highly expressed in many types of cancer, such as brain tumors [8], squamous cell carcinoma [23], soft tissue tumors [24], and bladder cancer [25]. RIT with ${ }^{225}$ Ac-labeled NZ16 is applicable for the treatment of such cancers, although further preclinical studies in these cancer models are required.

Our pathologic analysis showed that ${ }^{225}$ Ac-labeled NZ-16 induced a larger extent of necrotic change in tumor tissues compared with ${ }^{90}$ Y-labeled NZ-16, although the extent of apoptotic cell death and reduction of proliferating cells were similar. $\alpha$-Emitters can provide a large amount of energy and induce irreparable damage to cells, resulting in more cell death, manifested as apoptosis or necrosis, compared with $\beta$-emitters [10]. Our findings revealed that ${ }^{225}$ Ac-labeled NZ-16 more frequently induced necrosis than apoptosis. This result is consistent with previous reports of RIT with $\alpha$-emitters that apoptotic change is not often observed in solid tumors [26]. Further studies with various cancer types are needed to evaluate whether $\alpha-$ particle-induced cell death is depending on the cancer type.

In the present study, the estimated tumor absorbed doses following treatment with $3.7 \mathrm{MBq}$ of ${ }^{90}$ Y-labeled NZ-16 and $11.1 \mathrm{kBq}$ of ${ }^{225}$ Ac-labeled NZ-16 were 14.9 and 2.6 Gy, respectively (Table 4). Our finding indicates that the RBE of ${ }^{225} \mathrm{Ac}$-labeled NZ-16 is 5.7, which is similar to the recommended amount for $\alpha$-emitters by the Medical Internal Radiation Dose committee [22]. The treatment with ${ }^{225}$ Ac-labeled NZ-16 was markedly more effective than that with ${ }^{90} \mathrm{Y}-$ labeled NZ-16, suggesting that the real RBE is greater than 5.7. To estimate the RBE by another method, we referred to the results of X-ray treatment against $\mathrm{H} 226$ tumors in a previous report [15]. The efficacy of $11.1 \mathrm{kBq}$ of ${ }^{225}$ Ac-labeled NZ-16 was almost equivalent to that of $50 \mathrm{~Gy}$ of X-ray radiation, and the RBE was calculated to be 19.2 for ${ }^{225} \mathrm{Ac}$ relative to X-rays. The 
efficacy of $3.7 \mathrm{MBq}$ of ${ }^{90}$ Y-labeled NZ-16 was equivalent to 25 Gy of X-ray irradiation, and the RBE was calculated to be 1.7 for ${ }^{90} \mathrm{Y}$ relative to X-rays. Taken together, the RBE for ${ }^{225} \mathrm{Ac}$ to ${ }^{90} \mathrm{Y}$ was calculated to be 11.3 . The RBE values for the cell-killing effects of $\alpha$-emitters are reported to be 3 to 5 on the basis of in vivo experiments [22]. Our previous study with $\alpha$ emitting ${ }^{211}$ At-MABG also showed that the RBE was approximately 3 [26]. Compared with other $\alpha$-emitting compounds, the calculated RBE for ${ }^{225}$ Ac-labeled NZ-16 is quite high. The reason for this discrepancy is not clear at present, but the increased RBE might depend on the tumor type. Further studies are necessary to estimate a more accurate RBE for ${ }^{225} \mathrm{Ac}$-labeled NZ-16 to predict the therapeutic efficacy and safety in patients.

The clinical safety of RIT with NZ-16 must be evaluated. Bone marrow is generally the dose-limiting tissue in RIT. The limiting absorbed doses in bone marrow are 6-9 Sv in rodents and 4.5 Sv in humans $[21,27]$. We provided the estimated absorbed doses of ${ }^{90} \mathrm{Y}$ - and ${ }^{225} \mathrm{Ac}-$ labeled NZ-16, considering a radiation weighting factor of 1 for ${ }^{90} \mathrm{Y}$ and 5 for ${ }^{225} \mathrm{Ac}$ [22] in Table 5. The dose to the bone marrow was $8.1 \mathrm{~Sv}$ for $3.7 \mathrm{MBq}$ of ${ }^{90}$ Y-labeled NZ-16 and 7.0 $\mathrm{Sv}$ for $11.1 \mathrm{kBq}$ of ${ }^{225} \mathrm{Ac}$-labeled NZ-16. These doses would be acceptable in rodents; treatment-related mortality and toxicity to the bone marrow were not observed in the present study, although there was a temporary decrease in body weight. The doses, however, are greater than the limiting dose in humans of $4.5 \mathrm{~Sv}$. The injected dose to patients should thus be decreased. The biodistribution of drugs, including antibodies, however, is generally not identical between humans and animals. Clinical dosimetry studies are needed to determine a safe injected radioactive dose for humans.

In mesothelioma patients, most mesothelioma cells spread into the diaphragm, chest wall, and mediastinum; radiotherapy is therefore limited due to the high risk of injury to the lungs and surrounding organs [28]. Radiation pneumonitis is the most common toxicity in patients treated with radiation for cancers in the thorax [29]. The mean dose to the lungs for a $20 \%$ risk of radiation pneumonitis is $20 \mathrm{~Sv}$ [29]. Our dosimetry showed that absorbed doses to the murine lungs were $8.8 \mathrm{~Sv}$ for $3.7 \mathrm{MBq}$ of ${ }^{90}$ Y-labeled NZ-16 and 11.5 Sv for $18.5 \mathrm{kBq}$ of ${ }^{225} \mathrm{Ac}$-labeled NZ-16. The absorbed doses in the spleen, liver, and kidneys would also be tolerable because these doses were lower than the tolerated doses in humans [29]. Therefore, the risk of radiationinduced toxicity from RIT with radiolabeled NZ-16 is expected to be low. Indeed, there was no observed damage to the kidneys, lungs, and spleen after administering ${ }^{90} \mathrm{Y}-$ and ${ }^{225} \mathrm{Ac}-$ 
labeled NZ-16 in the pathologic analysis. As mentioned above, although the pharmacokinetics differ between humans and animals, the radiation doses absorbed by major organs, except for the bone marrow, were reasonably low. Most absorbed doses are expected to be tolerable in patients, although further dosimetry studies in mesothelioma patients are required.

As a SPECT imaging agent for dosimetry and treatment monitoring, ${ }^{111} \mathrm{In}$-labeled antibodies are a suitable surrogate for ${ }^{225} \mathrm{Ac}$-labeled antibodies [30]. The biodistribution of ${ }^{111}$ In- and ${ }^{225}$ Ac-labeled NZ-16 antibodies differed significantly in the present study. Uptake of ${ }^{225}$ Ac-labeled NZ-16 in most organs tended to be higher than that of ${ }^{111}$ In-labeled NZ-16. As mentioned above, the results of the present study indicated that the RBE of ${ }^{225}$ Ac-labeled NZ16 on tumor growth suppression was greater than 5 . Taken together, the dosimetry of ${ }^{225} \mathrm{Ac}-$ labeled NZ-16 could be underestimated by ${ }^{111}$ In-labeled surrogate imaging. The dose of ${ }^{225} \mathrm{Ac}-$ labeled NZ-16 might induce some unexpected damage. Unexpected toxicity was found in clinical studies with ${ }^{225}$ Ac-labeled PSMA-617 [31], although it is not an antibody. There are many reports of clinical trials with the ${ }^{225}$ Ac-labeled antibody lintuzumab for acute myeloid leukemia [32], but, to our knowledge, no reports about the dosimetry have been published. Several new clinical trials of $\alpha$-RIT for solid tumors were recently initiated $[33,34]$, but the dosimetry and normal organ toxicity have not yet been reported. Although the present study revealed no severe damage in mice treated with ${ }^{225}$ Ac-labeled NZ-16, further studies, including dosimetric assessments of toxicity in the therapeutic use of $\alpha$-RIT for solid tumors, are needed to determine the appropriate dose of ${ }^{225}$ Ac-labeled NZ-16 for first-in-human studies with high confidence.

The present study has several limitations. First, ${ }^{225}$ Ac-labeled NZ-16 did not achieve complete remission. Further strategies are needed to improve the antitumor effect. Fractionated therapy with ${ }^{225}$ Ac-labeled NZ-16 is promising. Two fractions of ${ }^{225} \mathrm{Ac}$-lintuzumab produced complete remission in patients with hematologic malignancies [35]. The therapeutic effects of RIT might be enhanced by combining them with chemotherapeutic agents. Pemetrexed as a first-line chemotherapy for mesothelioma has a radiosensitizing effect and might therefore be particularly effective with ${ }^{225}$ Ac-labeled NZ-16 [36]. Second, our dosimetry in mice cannot be directly applied to determine the appropriate dose for malignant mesothelioma patients. Clinical imaging studies with ${ }^{111}$ In-labeled NZ-16 are required to guarantee the safety of 
therapeutic treatments. These studies would promote the possible clinical application of ${ }^{225} \mathrm{Ac}-$ labeled NZ-16 for malignant mesothelioma.

In conclusion, the novel anti-PDPN antibody NZ-16 has a higher binding affinity and higher tumor uptake compared with the previous antibody NZ-12. Treatment with ${ }^{225} \mathrm{Ac}-$ labeled NZ-16 showed a potent antitumor effect without obvious adverse effects in a mesothelioma mouse model. RIT with ${ }^{225} \mathrm{Ac}$-labeled NZ-16 is a promising potential therapeutic option for malignant mesothelioma patients. Our findings are encouraging and warrant further studies toward clinical applications.

\section{Acknowledgments}

We thank Yuriko Ogawa, Naoko Kuroda, and Akihito Abe for technical assistance and the staff in the Laboratory Animal Sciences section for animal management.

\section{References}

1. Robinson BW, Lake RA. Advances in malignant mesothelioma. N Engl J Med. 2005;353:1591-603. doi:10.1056/NEJMra050152.

2. Kindler HL, Ismaila N, Armato SG, 3rd, Bueno R, Hesdorffer M, Jahan T, et al. Treatment of Malignant Pleural Mesothelioma: American Society of Clinical Oncology Clinical Practice Guideline. J Clin Oncol. 2018;36:1343-73. doi:10.1200/JCO.2017.76.6394.

3. Nishinaga Y, Sato K, Yasui H, Taki S, Takahashi K, Shimizu M, et al. Targeted Phototherapy for Malignant Pleural Mesothelioma: Near-Infrared Photoimmunotherapy Targeting Podoplanin. Cells. 2020;9. doi:10.3390/cells9041019.

4. Quintanilla M, Montero-Montero L, Renart J, Martín-Villar E. Podoplanin in Inflammation and Cancer. International Journal of Molecular Sciences. 2019;20:707.

5. Yuan P, Temam S, El-Naggar A, Zhou X, Liu DD, Lee JJ, et al. Overexpression of podoplanin in oral cancer and its association with poor clinical outcome. Cancer. 2006;107:563-9. doi:10.1002/cncr.22061.

6. Mishima K, Kato Y, Kaneko MK, Nishikawa R, Hirose T, Matsutani M. Increased expression of podoplanin in malignant astrocytic tumors as a novel molecular marker of malignant progression. Acta Neuropathol. 2006;111:483-8. doi:10.1007/s00401-006-0063-y.

7. Nakazawa Y, Sato S, Naito M, Kato Y, Mishima K, Arai H, et al. Tetraspanin family member CD9 inhibits Aggrus/podoplanin-induced platelet aggregation and suppresses 
pulmonary metastasis. Blood. 2008;112:1730-9. doi:10.1182/blood-2007-11-124693.

8. Chandramohan V, Bao X, Kato Kaneko M, Kato Y, Keir ST, Szafranski SE, et al. Recombinant anti-podoplanin (NZ-1) immunotoxin for the treatment of malignant brain tumors. Int J Cancer. 2013;132:2339-48. doi:10.1002/ijc.27919.

9. Abe S, Morita Y, Kaneko MK, Hanibuchi M, Tsujimoto Y, Goto H, et al. A novel targeting therapy of malignant mesothelioma using anti-podoplanin antibody. J Immunol. 2013;190:6239-49. doi:10.4049/jimmunol.1300448.

10. Larson SM, Carrasquillo JA, Cheung NK, Press OW. Radioimmunotherapy of human tumours. Nat Rev Cancer. 2015;15:347-60. doi:10.1038/nrc3925.

11. Gudkov SV, Shilyagina NY, Vodeneev VA, Zvyagin AV. Targeted Radionuclide Therapy of Human Tumors. Int J Mol Sci. 2015;17. doi:10.3390/ijms17010033.

12. Kratochwil C, Bruchertseifer F, Giesel FL, Weis M, Verburg FA, Mottaghy F, et al. ${ }^{225}$ Ac-PSMA-617 for PSMA-Targeted alpha-Radiation Therapy of Metastatic CastrationResistant Prostate Cancer. J Nucl Med. 2016;57:1941-4. doi:10.2967/jnumed.116.178673.

13. Tafreshi NK, Doligalski ML, Tichacek CJ, Pandya DN, Budzevich MM, El-Haddad G, et al. Development of Targeted Alpha Particle Therapy for Solid Tumors. Molecules. 2019;24. doi:10.3390/molecules24234314.

14. Miederer M, Scheinberg DA, McDevitt MR. Realizing the potential of the Actinium225 radionuclide generator in targeted alpha particle therapy applications. Adv Drug Deliv Rev. 2008;60:1371-82. doi:10.1016/j.addr.2008.04.009.

15. Sudo H, Tsuji AB, Sugyo A, Saga T, Kaneko MK, Kato Y, et al. Therapeutic efficacy evaluation of radioimmunotherapy with ${ }^{90}$ Y-labeled anti-podoplanin antibody NZ-12 for mesothelioma. Cancer Sci. 2019;110:1653-64. doi:10.1111/cas.13979.

16. Kaneko MK, Abe S, Ogasawara S, Fujii Y, Yamada S, Murata T, et al. Chimeric AntiHuman Podoplanin Antibody NZ-12 of Lambda Light Chain Exerts Higher AntibodyDependent Cellular Cytotoxicity and Complement-Dependent Cytotoxicity Compared with NZ-8 of Kappa Light Chain. Monoclon Antib Immunodiagn Immunother. 2017;36:25-9. doi:10.1089/mab.2016.0047.

17. Kato Y, Kaneko MK, Kuno A, Uchiyama N, Amano K, Chiba Y, et al. Inhibition of tumor cell-induced platelet aggregation using a novel anti-podoplanin antibody reacting with its platelet-aggregation-stimulating domain. Biochem Biophys Res Commun. 2006;349:13017. doi:10.1016/j.bbrc.2006.08.171.

18. Maguire WF, McDevitt MR, Smith-Jones PM, Scheinberg DA. Efficient 1-step radiolabeling of monoclonal antibodies to high specific activity with ${ }^{225} \mathrm{Ac}$ for alpha-particle radioimmunotherapy of cancer. J Nucl Med. 2014;55:1492-8. doi:10.2967/jnumed.114.138347. 
19. Yoshida C, Tsuji AB, Sudo H, Sugyo A, Kikuchi T, Koizumi M, et al. Therapeutic efficacy of c-kit-targeted radioimmunotherapy using ${ }^{90}$ Y-labeled anti-c-kit antibodies in a mouse model of small cell lung cancer. PLoS One. 2013;8:e59248. doi:10.1371/journal.pone.0059248.

20. Eckerman KF, Endo A, MIRD radionuclide data and decay schemes. 2nd edition. Reston, VA: Society of Nuclear Medicine; 2007.

21. Behr TM, Sgouros G, Stabin MG, Behe M, Angerstein C, Blumenthal RD, et al. Studies on the red marrow dosimetry in radioimmunotherapy: an experimental investigation of factors influencing the radiation-induced myelotoxicity in therapy with beta-, Auger/conversion electron-, or alpha-emitters. Clin Cancer Res. 1999;5:3031s-43s.

22. Sgouros G, Roeske JC, McDevitt MR, Palm S, Allen BJ, Fisher DR, et al. MIRD Pamphlet No. 22 (Abridged): Radiobiology and Dosimetry of $\alpha$-Particle Emitters for Targeted Radionuclide Therapy. Journal of Nuclear Medicine. 2010;51:311-28. doi:10.2967/jnumed.108.058651.

23. Kato Y, Kaneko M, Sata M, Fujita N, Tsuruo T, Osawa M. Enhanced expression of Aggrus (T1alpha/podoplanin), a platelet-aggregation-inducing factor in lung squamous cell carcinoma. Tumour Biol. 2005;26:195-200. doi:10.1159/000086952.

24. Xu Y, Ogose A, Kawashima H, Hotta T, Ariizumi T, Li G, et al. High-level expression of podoplanin in benign and malignant soft tissue tumors: immunohistochemical and quantitative real-time RT-PCR analysis. Oncol Rep. 2011;25:599-607. doi:10.3892/or.2011.1141.

25. Kato Y, Sasagawa I, Kaneko M, Osawa M, Fujita N, Tsuruo T. Aggrus: a diagnostic marker that distinguishes seminoma from embryonal carcinoma in testicular germ cell tumors. Oncogene. 2004;23:8552-6. doi:10.1038/sj.onc.1207869.

26. Ohshima Y, Sudo H, Watanabe S, Nagatsu K, Tsuji AB, Sakashita T, et al. Antitumor effects of radionuclide treatment using alpha-emitting meta- ${ }^{211}$ At-astato-benzylguanidine in a PC12 pheochromocytoma model. Eur J Nucl Med Mol Imaging. 2018;45:999-1010. doi:10.1007/s00259-017-3919-6.

27. Frampas E, Maurel C, Remaud-Le Saec P, Mauxion T, Faivre-Chauvet A, Davodeau F, et al. Pretargeted radioimmunotherapy of colorectal cancer metastases: models and pharmacokinetics predict influence of the physical and radiochemical properties of the radionuclide. Eur J Nucl Med Mol Imaging. 2011;38:2153-64. doi:10.1007/s00259-011-19030.

28. Nadal E, Bosch-Barrera J, Cedres S, Coves J, Garcia-Campelo R, Guirado M, et al. SEOM clinical guidelines for the treatment of malignant pleural mesothelioma (2020). Clin 
Transl Oncol. 2021;23:980-7. doi:10.1007/s12094-020-02532-2.

29. Emami B. Tolerance of Normal Tissue to Therapeutic Radiation. Rep Radiother Oncol. 2013;1:123-7.

30. Nedrow JR, Josefsson A, Park S, Back T, Hobbs RF, Brayton C, et al. Pharmacokinetics, microscale distribution, and dosimetry of alpha-emitter-labeled anti-PD-L1 antibodies in an immune competent transgenic breast cancer model. EJNMMI Res. 2017;7:57. doi:10.1186/s13550-017-0303-2.

31. Kratochwil C, Bruchertseifer F, Rathke H, Bronzel M, Apostolidis C, Weichert W, et al. Targeted $\alpha$-Therapy of Metastatic Castration-Resistant Prostate Cancer with ${ }^{225}$ Ac-PSMA617: Dosimetry Estimate and Empiric Dose Finding. J Nucl Med. 2017;58:1624-31. doi:10.2967/jnumed.117.191395.

32. Jurcic J, Levy M, Park J, Ravandi F, Perl A, Pagel J, et al. Phase I trial of alpha-particle immunotherapy with ${ }^{225} \mathrm{Ac}$-lintuzumab and low-dose cytarabine in patients age 60 or older with untreated acute myeloid leukemia. Journal of Nuclear Medicine. 2017;58:456-.

33. Juergens RA, Zukotynski KA, Juneau D, Krnezich L, Simms R, Forbes J, et al. A phase I study of $\left[{ }^{225} \mathrm{Ac}\right]-\mathrm{FPI}-1434$ radioimmunotherapy in patients with IGF-1R expressing solid tumors. Journal of Clinical Oncology. 2019;37:TPS3152-TPS. doi:10.1200/JCO.2019.37.15_suppl.TPS3152.

34. Tagawa ST, Osborne J, Niaz MJ, Vallabhajosula S, Vlachostergios PJ, Thomas C, et al. Dose-escalation results of a phase I study of ${ }^{225} \mathrm{Ac}-\mathrm{J} 591$ for progressive metastatic castration resistant prostate cancer (mCRPC). Journal of Clinical Oncology. 2020;38:114-. doi:10.1200/JCO.2020.38.6_suppl.114.

35. Jurcic JG. Targeted Alpha-Particle Therapy for Hematologic Malignancies. J Med Imaging Radiat Sci. 2019;50:S53-s7. doi:10.1016/j.jmir.2019.05.008.

36. Oleinick NL, Biswas T, Patel R, Tao M, Patel R, Weeks L, et al. Radiosensitization of non-small-cell lung cancer cells and xenografts by the interactive effects of pemetrexed and methoxyamine. Radiother Oncol. 2016;121:335-41. doi:10.1016/j.radonc.2016.10.007.

\section{Figure legends}

Figure 1. In vitro characterization of radiolabeled antibodies NZ-12 and NZ-16. (a) Cell binding assay of ${ }^{111}$ In-labeled NZ-12 with H226 cells. (b) Cell binding assay of ${ }^{111}$ In-labeled NZ-16 with H226 cells. (c) Competitive inhibition assay for intact NZ-12 (white circles) and 
DOTA-conjugated NZ-12 (black circles) with H226 cells. (d) Competitive inhibition assay for intact NZ-16 (white circles) and DOTA-conjugated NZ-16 (black circles) with H226 cells.

Figure 2. Tumor uptake of ${ }^{111} \mathrm{In}$-labeled NZ-12, and ${ }^{111} \mathrm{In}-$ and ${ }^{225} \mathrm{Ac}-\mathrm{labeled} \mathrm{NZ-16}$. Data indicate the mean and standard deviation $(\mathrm{n}=4-5) . * * P<0.01$

Figure 3. Therapeutic efficacy of ${ }^{90} \mathrm{Y}$ - and ${ }^{225}$ Ac-labeled antibodies in $\mathrm{H} 226$ tumor-bearing mice. (a) Tumor growth curves of individual mice after injection with ${ }^{90} \mathrm{Y}$ - or ${ }^{225} \mathrm{Ac}-\mathrm{labeled}$ NZ-16 (thin lines). Bold lines indicate the mean. (b) Kaplan-Meier survival curves based on a tumor volume endpoint of $300 \mathrm{~mm}^{3} . * * P<0.01$, vs. $0 \mathrm{MBq} \mathrm{NZ}-16$.

Figure 4. Hematoxylin and eosin-stained sections of H226 tumors treated with $0 \mathrm{MBq}$ (intact NZ-16 only), 3.7 MBq of ${ }^{90}$ Y-labeled NZ-16, and $18.5 \mathrm{kBq}$ of ${ }^{225}$ Ac-labeled NZ-16 at days 1, 3, and 7 after injection. Arrowheads indicate necrosis. N, necrosis; T, tumor; F, fibrous tissue; bars, $50 \mu \mathrm{m}$

Figure 5. Tumor cell proliferation analysis using Ki-67 immunostaining. (a) Ki-67-stained $\mathrm{H} 226$ tumors at days 1,3 , and 7 after injection with $0 \mathrm{MBq}$ (intact NZ-16 only), 3.7 MBq of ${ }^{90}$ Y-labeled NZ-16, and $18.5 \mathrm{MBq}{ }^{225}$ Ac-labeled NZ-16. Bar, $50 \mu \mathrm{m}$ (b) Quantification of proliferating (Ki-67 positive) cells. Data represent the mean and standard deviation. ${ }^{*} P<0.01$. 
Figures
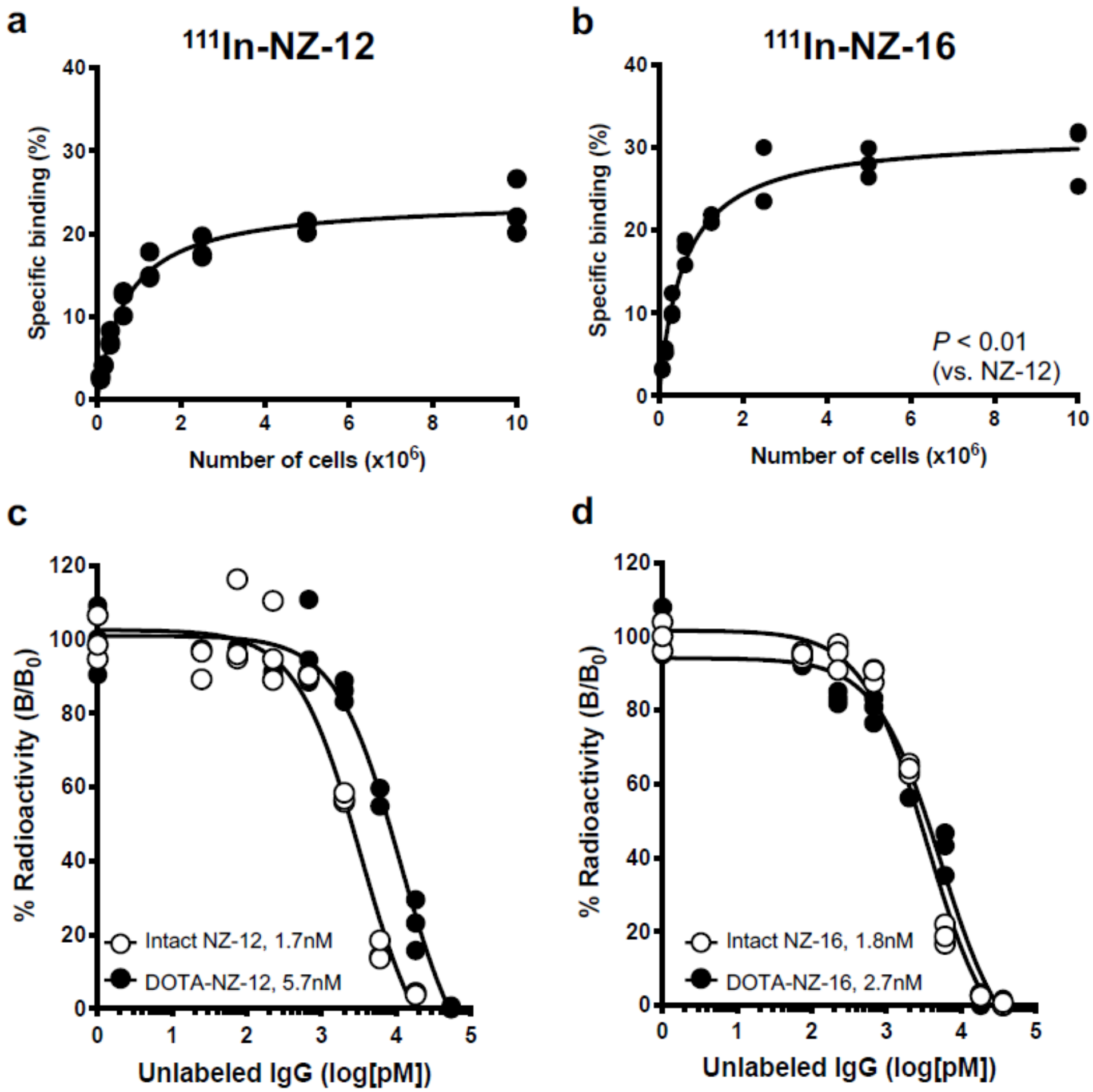

Figure 1

In vitro characterization of radiolabeled antibody NZ- 12 and NZ-16. (a) Cell binding assay of $111 \mathrm{ln}$ labeled NZ-12 with H226 cells. (b) Cell binding assay of 111 In-labeled NZ-16 with H226 cells. (c) Competitive inhibition assay for intact NZ-12 (white circles) and DOTA-conjugated NZ-12 (black circles) 
with $\mathrm{H} 226$ cells. (d) Competitive inhibition assay for intact NZ-16 (white circles) and DOTA-conjugated NZ-16 (black circles) with H226 cells.

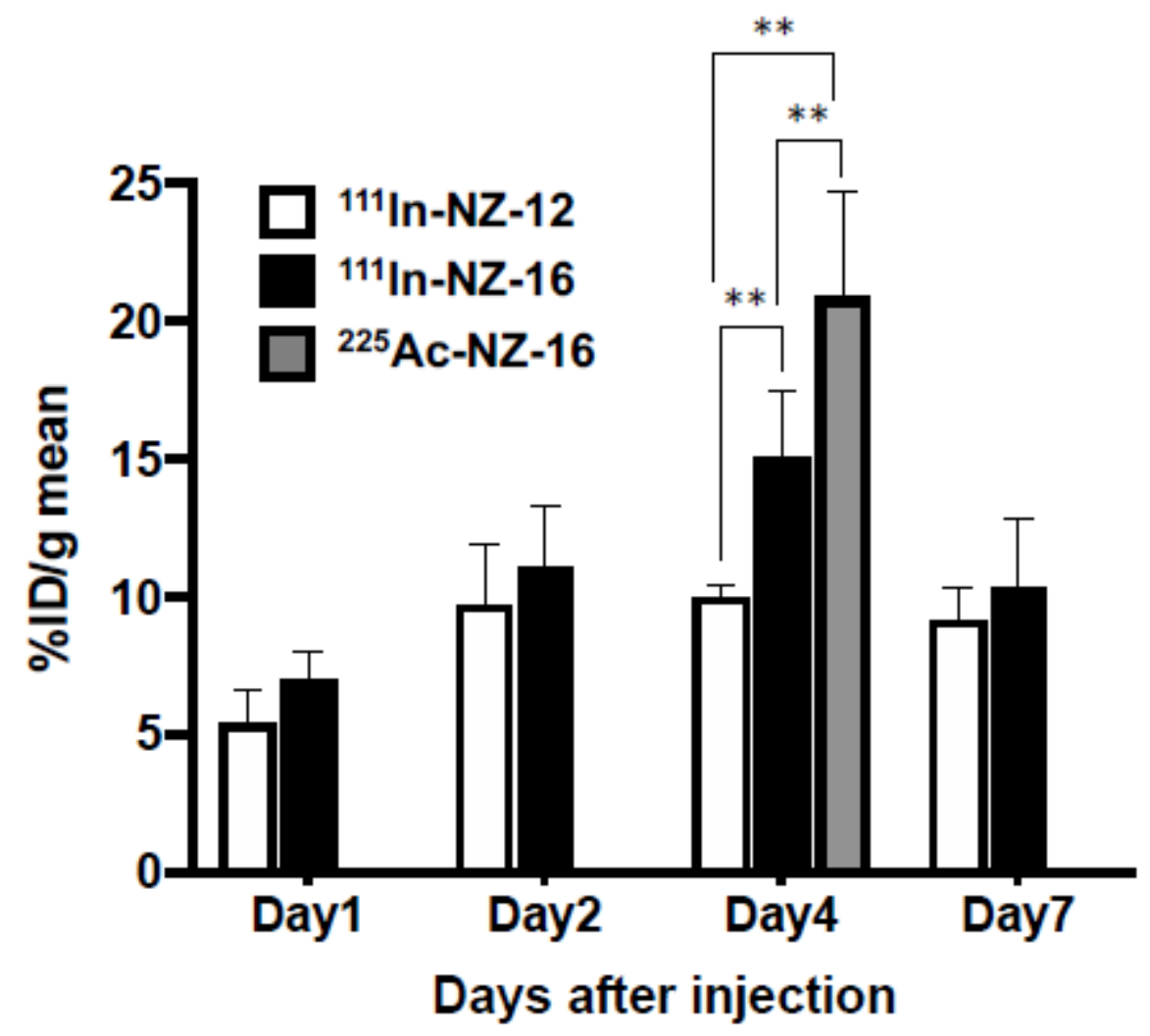

Figure 2

Tumor uptake of 111 Inlabeled NZ-12, and 111 In- and 225Aclabeled NZ-16. Data indicate mean and standard deviation $(n=4-5) .{ }^{* \star P}<0.01$ 
a
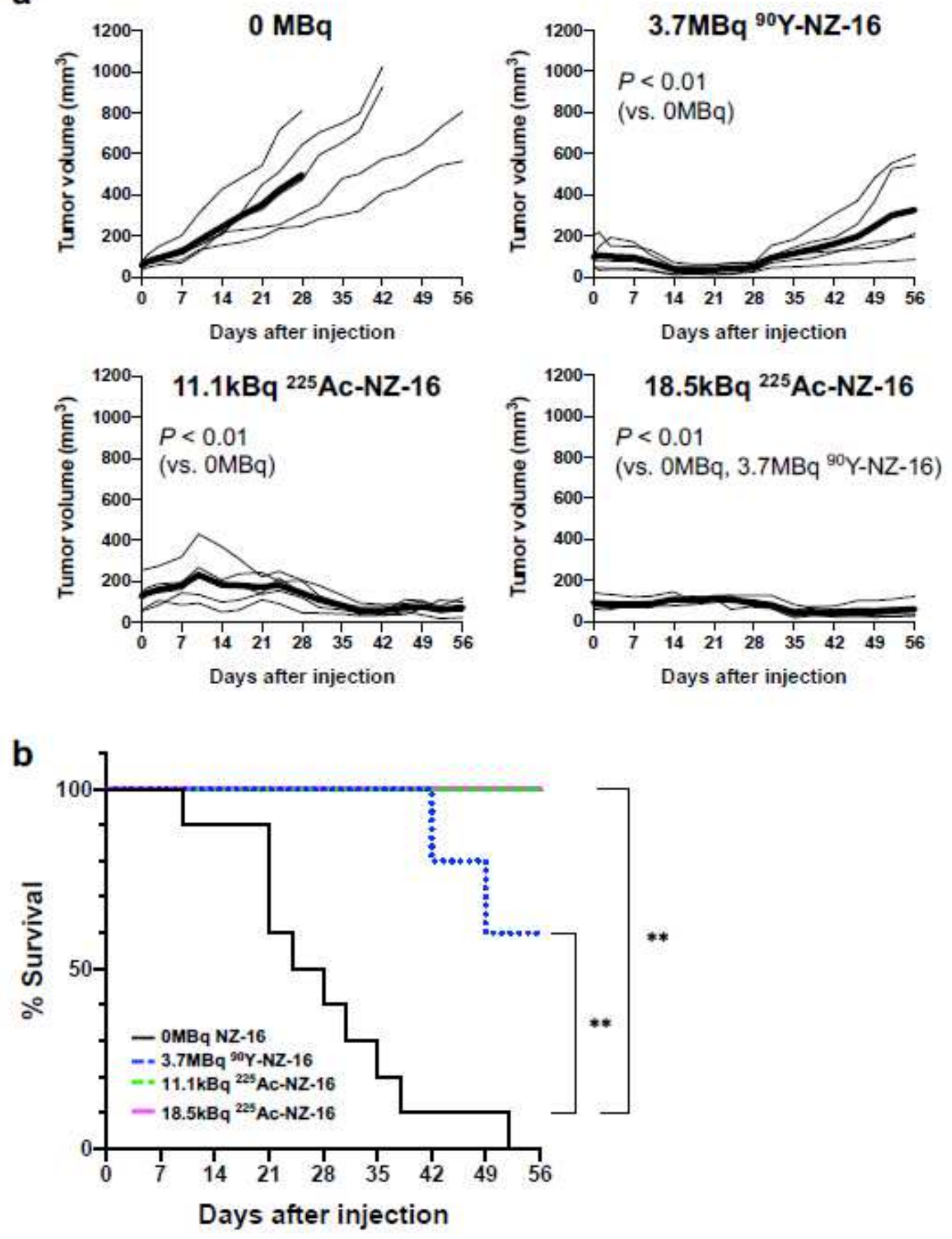

\section{Figure 3}

Therapeutic efficacy of 90Y-and 225Ac-labeled antibodies in H226 tumor-bearing mice. (a) Tumor growth curves of each mouse after injection with 90Y- or 225Ac-labeled NZ-16 (thin lines). Bold lines indicate mean. (b) Kaplan-Meier survival curves based on the endpoints of tumor volume of $300 \mathrm{~mm} 3 . * \star \mathrm{P}<0.01$, vs. 0 MBq NZ-16. 


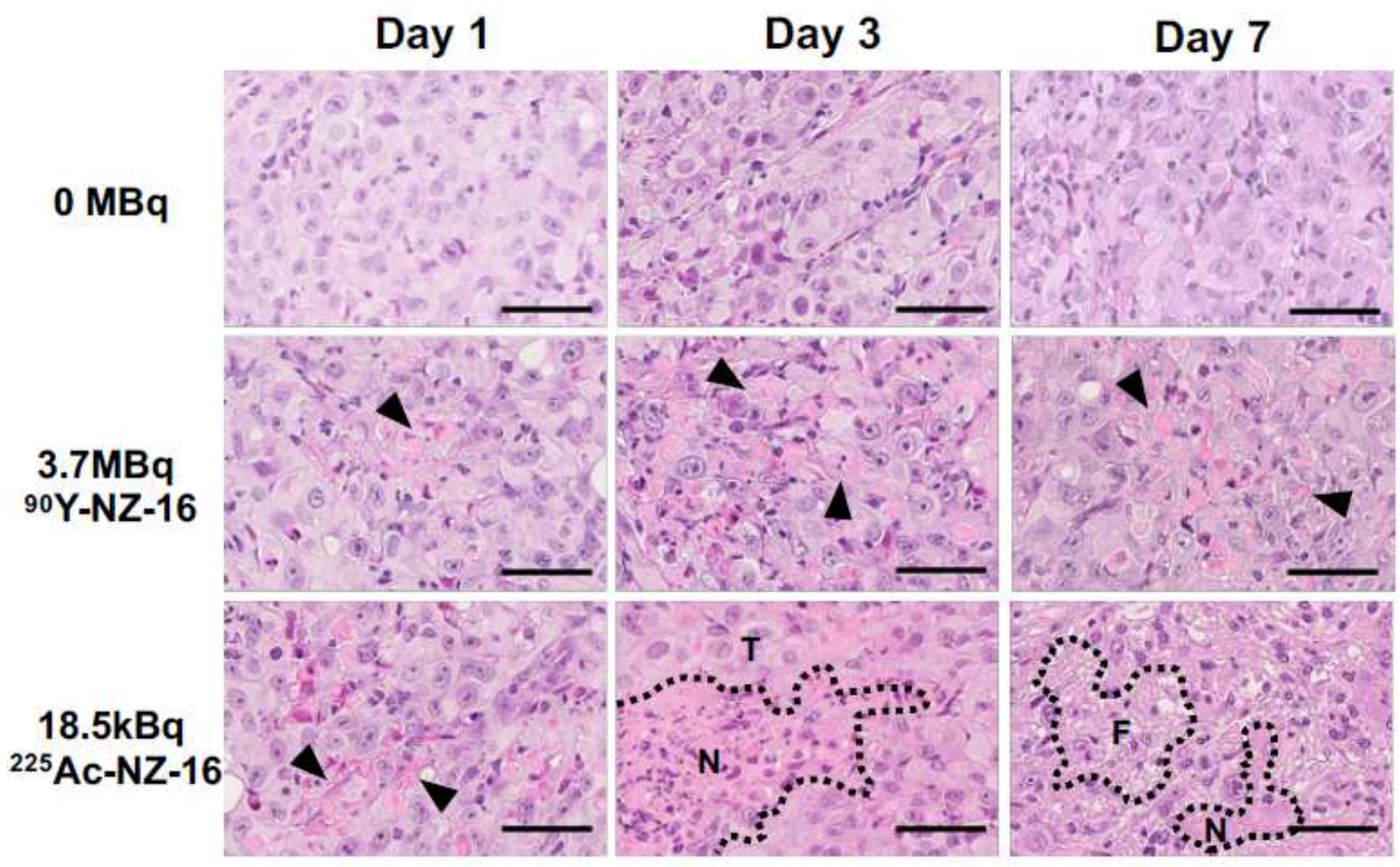

Figure 4

H\&E-stained sections of H226 tumors treated with $0 \mathrm{MBq}$ (intact NZ-16 only), 3.7 MBq of 90Y-labeled NZ16 , and $18.5 \mathrm{kBq}$ of $225 \mathrm{Ac}-\mathrm{labeled} \mathrm{NZ}-16$ at days 1,3 , and 7 after injection. Arrowheads indicate necrosis. $\mathrm{N}$, necrosis; T, tumor; F, fibrous tissue; bars, $50 \mu \mathrm{m}$ 
a

Day 1 Day 3 Day 7

OMBq

$3.7 \mathrm{MBq}$ ${ }^{90}$ Y-NZ-16

$18.5 \mathrm{kBq}$ ${ }^{225} \mathrm{Ac}-\mathrm{NZ}-16$
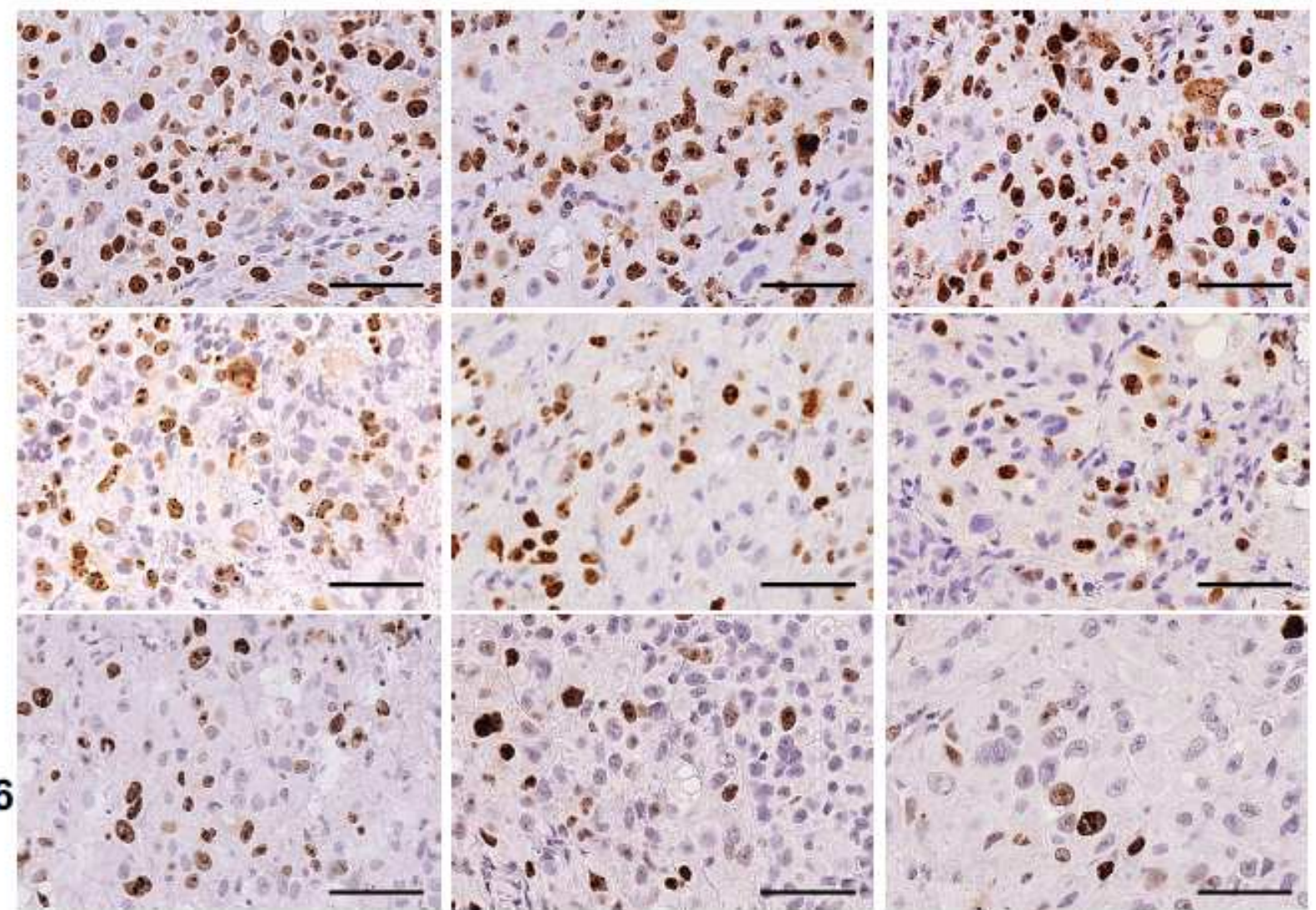

b

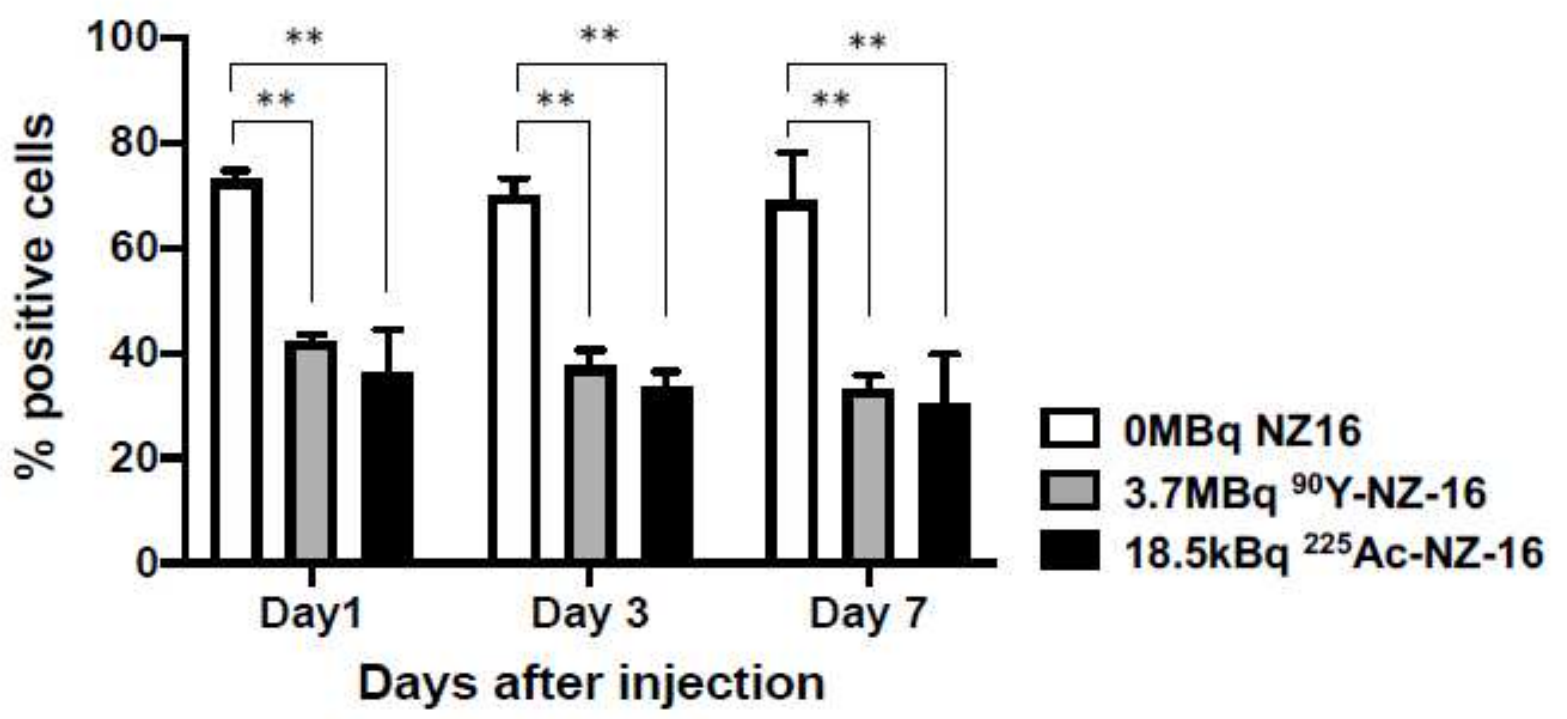

Figure 5

Tumor cell proliferation analysis using Ki-67 immunostaining. (a) Ki-67-stained H226 tumors at days 1, 3, and 7 after injection with $0 \mathrm{MBq}$ (intact NZ-16 only), 3.7 MBq of 90Ylabeled NZ-16, and 225Ac-labeled NZ-16. Bar, $50 \mu \mathrm{m}$ (b) Quantification of proliferating (Ki-67 positive) cells. Data represent the mean and standard deviation. ${ }^{\star \star} \mathrm{P}<0.01$. 


\section{Supplementary Files}

This is a list of supplementary files associated with this preprint. Click to download.

- PDPNTable210706.pdf

- PDPNSupple210706.pdf 